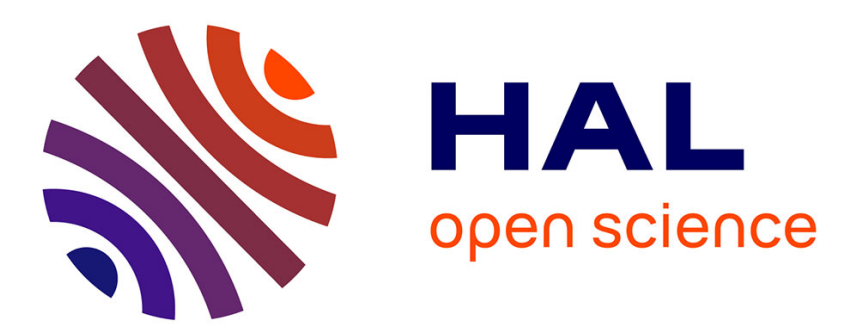

\title{
Uncertainty of reference frames characterized by real time optical measurements: Application to Computer Assisted Orthopaedic Surgery
}

Jean-Marc Linares, Jean-Michel Sprauel, P. Bourdet

\section{To cite this version:}

Jean-Marc Linares, Jean-Michel Sprauel, P. Bourdet. Uncertainty of reference frames characterized by real time optical measurements: Application to Computer Assisted Orthopaedic Surgery. CIRP Annals - Manufacturing Technology, 2009, 58 (1), pp.447 - 450. 10.1016/j.cirp.2009.03.008 . hal01426919

HAL Id: hal-01426919

https://hal-amu.archives-ouvertes.fr/hal-01426919

Submitted on 5 Jan 2017

HAL is a multi-disciplinary open access archive for the deposit and dissemination of scientific research documents, whether they are published or not. The documents may come from teaching and research institutions in France or abroad, or from public or private research centers.
L'archive ouverte pluridisciplinaire HAL, est destinée au dépôt et à la diffusion de documents scientifiques de niveau recherche, publiés ou non, émanant des établissements d'enseignement et de recherche français ou étrangers, des laboratoires publics ou privés. 


\title{
Uncertainty of reference frames characterized by real time optical measurements: Application to Computer Assisted Orthopaedic Surgery
}

\author{
J.M. Linares ${ }^{\mathrm{a}, *}$, J.M. Sprauel ${ }^{\mathrm{a}}$, P. Bourdet $(1)^{\mathrm{b}}$

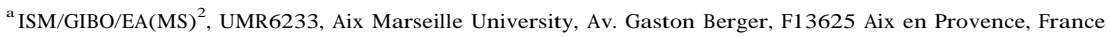 \\ ${ }^{b}$ E.N.S Cachan, 61 Av. President Wilson, 94230 Cachan, France
}

In total knee arthroplasty (TKA), real time optical measurements are now carried out to improve the implantation of prostheses The final alignment of prostheses is however influenced by the choice of points probed by the surgeon, and by the measurement accuracy. A Monte Carlo method has been developed, to simulate a whole set of measurements. To estimate the uncertainties of the resultant angles between the two parts of the knee prosthesis, this data has then been treated with the algorithms implemented in Computer Assisted Orthopaedic Surgery programs. Such results were also obtained through uncertainty propagations based on the GUM.

Keywords: Uncertainty Geometric modelling, Computer Assisted Orthopaedic, Surgery (CAOS)

\section{Introduction}

Healthcare is one of the fastest growing western markets [1]. In this topic, the implantation of prostheses represents a nonnegligible share of health costs. Total knee arthroplasty (TKA) is one of the most common orthopaedic surgeries. Currently, premature failure of the prosthesis is often related to an excess varus tibial bone cut. Computer Assisted Orthopaedic Surgery (CAOS) has therefore been developed to improve the implantation process [2].

For that purpose the cutting of biomaterials has first been studied and improved [3,4]. Automated optical measurements are already applied, now, in arthroplasty protocols to control the geometrical quality of the implantation process [2]. Multi-axis CAM systems are also developed for the same purpose [2,5].

However, even with such advancements, significant angular dispersions are observed by surgeons in the results of arthroplasty. It is therefore important to control the dispersions of the anatomic angles (a: flexion-extension, $\mathbf{b}$ : varus-valgus and $\mathrm{g}$ : axial rotation angles) observed after implantation (Fig. 2).

Fig. 1 summarizes the different sources of these angular errors and uncertainties. Our study will focus on the two major factors of uncertainty: the accuracy of the optical measurements and the dispersion of the anatomic landmarks selected by the surgeon. However, the procedures, imposed by the CAOS system, cannot be repeated on a real patient. Different solutions are nevertheless available, in applications of mechanical engineering to define uncertainties. These methods are based either on Monte Carlo simulation methods [6,7] or analytical propagation techniques based on the Guide to the Expression of Uncertainty in Measurement (GUM) $[8,9]$. They will be applied now to TKA process.

\section{Geometrical model}

The optimal implantation of the prosthesis is based on an accurate determination of the mechanical anatomic axes of the tibia and the femur.

Coordinate systems are thus constructed on both bones, based on the acquisition of specific landmarks found by identification of the desired points by visual selection and by palpation (Fig. 2). They include the centre of the femoral head $\left(\mathrm{O}_{\text {hip }}\right)$ defined by a kinematics method, the centre of distal femur $\left(\mathrm{O}_{\mathrm{Femur}}\right)$, the medial $\left(\mathrm{C}_{1}\right)$ and lateral $\left(\mathrm{C}_{2}\right)$ femoral epicondyle, the centre of proximal tibia $\left(\mathrm{O}_{\text {Tibia }}\right)$, the normal direction $\left(\mathrm{V}_{\mathrm{ATT}}\right)$ of the anterior tibial tubirosity (ATT), medial malleolus $\left(\mathrm{M}_{1}\right)$, and lateral malleolus $\left(\mathrm{M}_{2}\right)$. Since the patient may slightly move during TKA process, optical reference frames are fixed on the femur and the tibia to characterize theses displacement in real time.

\section{Measurement simulation}

TKA process cannot be repeated on one single patient. A virtual parametric model of the measurements carried out during TKA process has therefore been developed to estimate the uncertainties. The geometry of the simulation model is described by a parametric skeleton characterizing the mechanical axes of the bones and their anatomic landmarks, and the optical reference frames fixed to the tibia and the femur (Fig. 3).

These last are defined by measured points $\left(\mathrm{P}_{1}, \mathrm{P}_{2}, \mathrm{P}_{3}\right)$ and $\left(\mathrm{Q}_{1}, \mathrm{Q}_{2}, \mathrm{Q}_{3}\right)$. The coordinates of the anatomic landmarks are derived from the points $\left(\mathrm{N}_{1}, \mathrm{~N}_{2}, \mathrm{~N}_{3}\right)$ of a ball tip pointer. The model allows setting any virtual configurations of TKA process, just changing the input parameters (angles) of the skeleton. This permits simulating the position of the points $\mathrm{P}_{\mathrm{i}}, \mathrm{Q}_{\mathrm{i}}$, and $\mathrm{N}_{\mathrm{i}}$ which are the digitalized coordinates entered in the real CAOS system. 


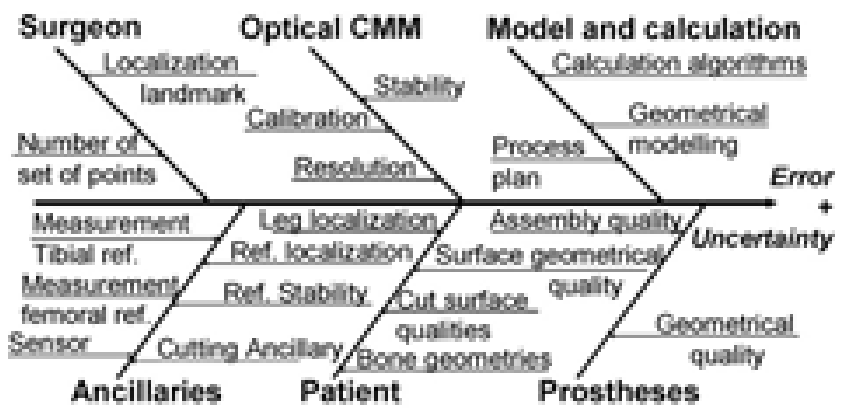

Fig. 1. Error and uncertainty.

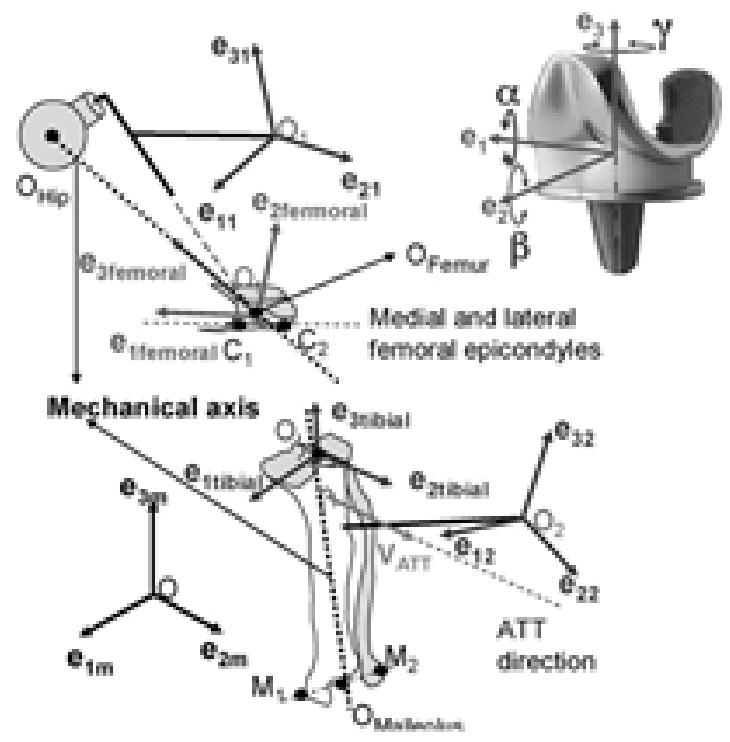

Fig. 2. Geometrical modelling of TKA.

4. Basic procedures of TKA CAOS system

Our study required first rewriting the basic procedures implemented in CAOS system software. The first step of the process plan of TKA is to define the hip centre. This operation is realized through a kinematics method (Fig. 4).

Since points $\mathrm{P}_{\mathrm{i}}$ are thus moving on spheres, the mean values and the covariance matrix of hip centre coordinates are derived by a nonlinear least squares method.

Another basic procedure is also required to derive the coordinates $\mathrm{M}_{\mathrm{i}}$ of each probed landmark from the set of points $\mathrm{N}_{\mathrm{i}}$ acquired by the ball tip pointer. These coordinates are to be

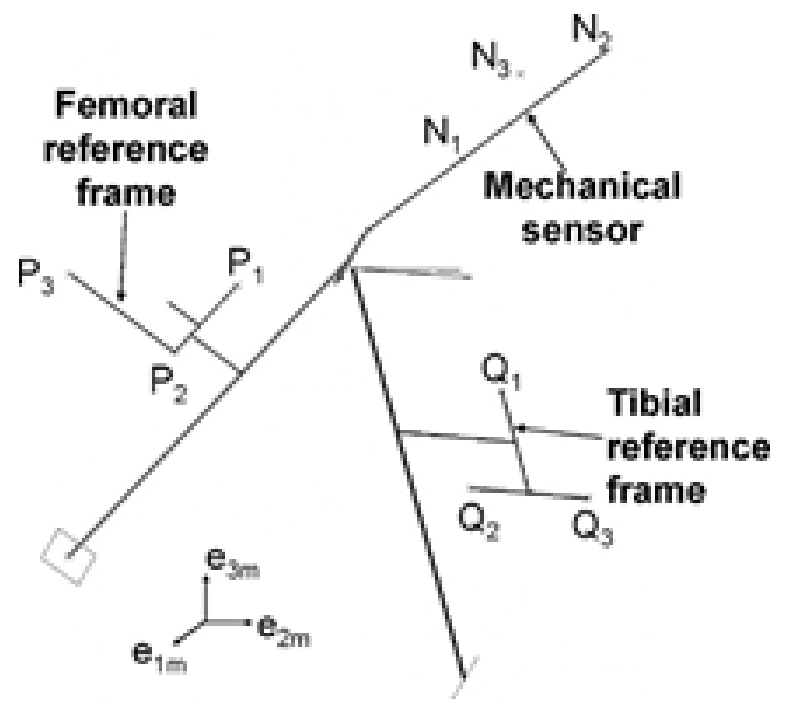

Fig. 3. Virtual parametric model skeleton.
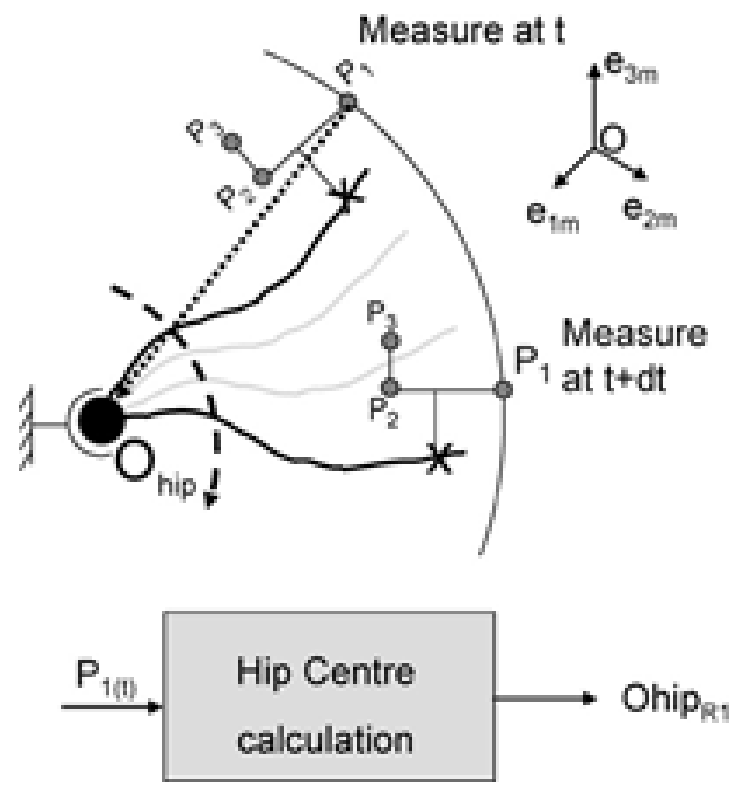

Fig. 4. Measure and calculation of hip centre.

expressed in the local coordinate systems linked to each bone. It requires a real time acquisition of the optical reference frames points $P_{i}$ or $Q_{i}$, in order to account for the movements of the patient The ball tip calibration lengths are also to be known. The input factors of this calculus are shown in Fig. 5.

The same kind of approach is also used to determine the ATT direction (Fig. 6).

All these calculation procedures allow constructing, at any time, coordinate systems characterizing the absolute positions and
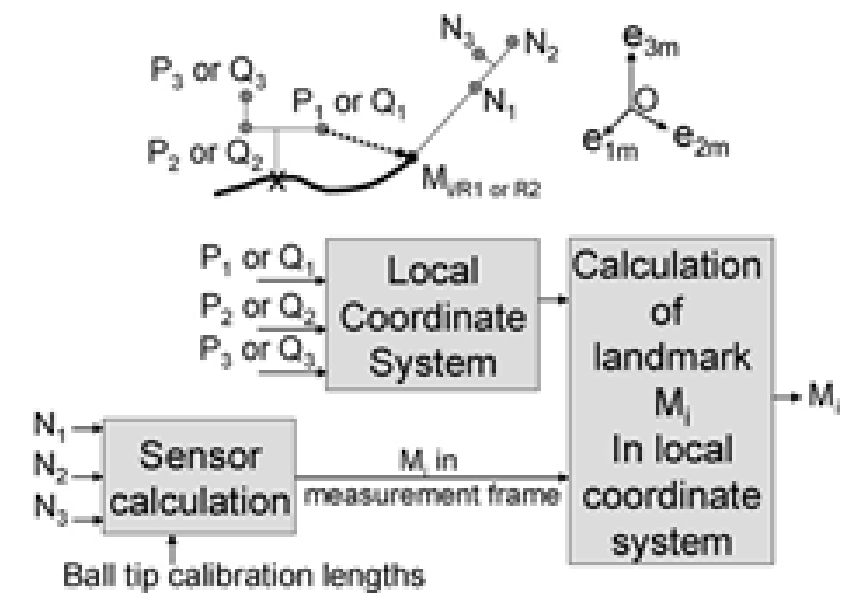

Fig. 5. Measured input points used to derive the real time coordinates $M_{i}$ of a probed landmark.

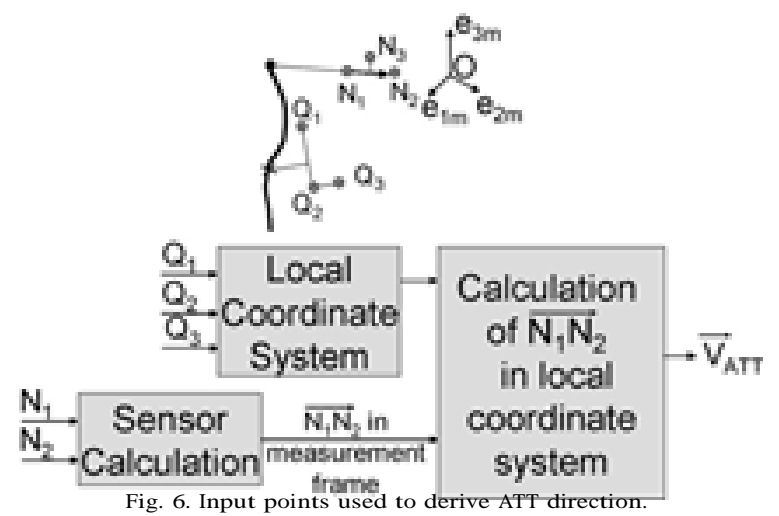


orientations of the femur and the tibia. This permits defining the cutting planes for arthroplasty, whose variations are the main source of uncertainty of the final adjustment of the prosthesis.

\section{Uncertainty evaluation: Monte Carlo simulation and} analytical propagation

Previous calculation procedures were implemented and applied to the whole process plan of TKA, in order to compute the uncertainties of the angles between the two parts of the prosthesis. This paper will focus on the two major factors of uncertainty: the accuracy of the optical measurements and the dispersion of the anatomic landmarks selected by the surgeon. For all points, the measurements are assumed to be uniformly scattered in a spherical localisation zone. The perturbations are thus assumed to have the same amplitude in any direction. Consequently, the covariance matrix of each basic measurement takes a spherical form.

\subsection{Monte Carlo simulation}

Monte Carlo simulation is a common tool in uncertainty evaluation of complex measurement processes. It is used because of the lack or the difficulty to express analytical solutions. In our study, this method was employed to calculate the mean values and the covariance matrixes of the angles between the two parts of the prosthesis, i.e. the flexion angle $\mathbf{a}$, the valgus-varus angle $\mathbf{b}$ and the axial rotation angle $\mathrm{g}$. For that purpose, the three coordinates of each measured point are first generated randomly using the specific implementation which will be presented below. About hundred points $\left(\mathrm{P}_{\mathrm{i}}, \mathrm{Q}_{\mathrm{i}}, \mathrm{N}_{\mathrm{i}}\right)$ are thus built for each simulated TKA process. Second, the angles $(\mathbf{a}, \mathbf{b}, \mathbf{g})$ between the two parts of the prosthesis are deduced from this whole set of data, using geometrical calculations. From a great number of simulations, their mean values and covariances are finally computed through classical statistical analysis.

Monte Carlo simulation is very simple to implement. The method requires however all input variables to be independent. A classical Cartesian representation of the deviations to the mean coordinates cannot thus be used to simulate a uniform random repartition of measured points in a spherical localisation zone. A spherical coordinate system parameterization $\left(\mathrm{U}_{\mathrm{i}}, \mathrm{w}_{\mathrm{i}}, \mathbf{r}_{\mathrm{i}}\right)$ of the deviations is thus useful to solve the problem. An accurate simulation of measurement data needs however accounting for the non-uniform distribution of the independent variables $\left(u_{i}, w_{i}, r_{i}\right)$. This leads to following random generation equations:

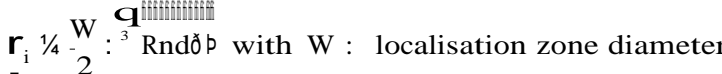

$$
\begin{aligned}
& \text {, } 1 / 4 \text { Arccosð } 1 \ddot{\text { y } 2: R n d ð p p ~} \\
& \mathrm{u}_{\mathrm{i}} 1 / 4 \text { 2:P:Rndð } \mathrm{P}
\end{aligned}
$$

In these equations, Rnd( ) is the random generation of a uniformly distributed real variable in the interval $[0,1]$. Mersenne-Twister pseudo-random generation algorithm has been used to avoid sequential correlations between the different independent variables.

The convergence rate of the Monte Carlo method is $\mathrm{O} \delta 1={ }_{\mathrm{N}}^{\mathrm{N}} \mathrm{N}$. where $\mathrm{N}$ is the number of simulated experiments. For each simulation, at least 30,000 sets of points $\left(\mathrm{P}_{\mathrm{i}}, \mathrm{Q}_{\mathrm{i}}, \mathrm{N}_{\mathrm{i}}\right)$ were therefore built randomly in order to limit the imprecision of the final results.

\subsection{Analytical propagation}

In this method, the uncertainties are defined by the classical expression of the GUM [9]:

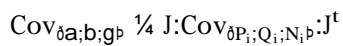

Each component of the Jacobian matrix $\mathrm{J}$ is the partial derivative of angle $\mathbf{a}, \mathbf{b}$ or $\mathbf{g}$ to a given coordinate of the numerous measured points $\left(\mathrm{P}_{\mathrm{i}}, \mathrm{Q}_{\mathrm{i}}, \mathrm{N}_{\mathrm{i}}\right)$. In a complex process, like our problem, it is thus very fastidious to calculate the Jacobian matrix of the functions of interest. A numerical calculation of the partial derivatives was therefore developed and implemented. It is based on a third degree polynomial approximation of the evolution of the angles $\mathbf{a}, \mathbf{b}$ and $g$ versus each input parameter. These angles were then computed for four values (evenly distributed in the localisation interval) of a given input coordinate, keeping all other variables fixed to their mean value. This allowed straight evaluation of the coefficients of each approximation polynomial and finally of the components of the Jacobian matrix.

The measured coordinates are assumed to be independent one

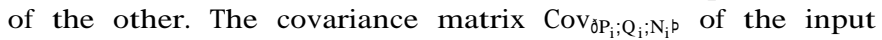
parameters takes therefore a diagonal form. It consists of blocks of elementary diagonal spherical covariance matrixes characterizing the scattering of the acquisitions carried out on individual points. Since each measured point is assumed to be uniformly distributed in a spherical localisation zone, the standard deviation $S$ of its coordinates is directly linked to the diameter W of its boundary:

$$
\text { S 1/4 } \frac{\text { W }}{{ }^{\text {Pling }}}
$$

For the kinematics method employed to define the hip centre, the diameter W just characterizes the inaccuracy of the optical CMM. During acquisition of anatomic landmarks, it also incorporates the scattering of the points probed by the surgeon.

\section{Results}

Both Monte Carlo simulation method and the analytical propagation technique were applied to a typical configuration of TKA. The manufacturer of the CMM certifies that the dispersions of the optical measurements are limited to $0.1 \mathrm{~mm}$. However, in order to separate the contributions of the two major sources of dispersion, the calculations were performed for uncertainty zones of $\mathrm{W}=0.05,0.1$ and $0.15 \mathrm{~mm}$. For each value, the scattering of the landmarks probed by the surgeon was varying from 0.5 to $5 \mathrm{~mm}$.

The expanded uncertainty $\mathrm{U}(\mathrm{k}=2)$ of the different angles between the two parts of the prosthesis were then derived from the covariance matrixes obtained either by Monte Carlo simulation or analytical propagation. The two methods lead to similar results. Fig. 7 shows the curves obtained by Monte Carlo simulation. The results calculated through analytical propagation are presented in Fig. 8. In these plots, the curves drawn for the three different resolutions of the optical measurements are almost superimposed. It demonstrates the low impact of this source of uncertainty on the final adjustment of the prosthesis.

On the contrary, the uncertainties of the different angles are strongly amplified with increasing scattering of the landmarks probed by the surgeon. The flexion angle $\mathrm{g}$ is most impacted by this inaccuracy whereas the error bars on the flexion angle $\mathbf{a}$ and the valgus-varus angle $b$ remain low.

By principle, the mean angles calculated by the analytical method do not depend on the amplitude of the measurement uncertainties, since they derive directly from the mean coordinates of the acquired points. On the contrary, as shown in Fig. 9, the Monte Carlo method shows small bias. This may be due to the

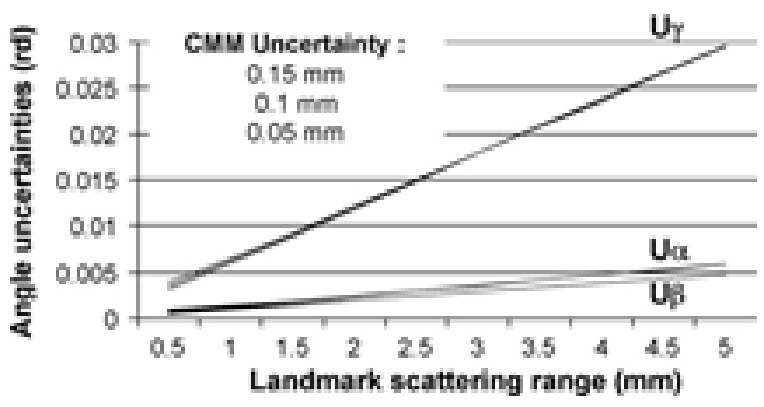

Fig. 7. Uncertainties evaluated by Monte Carlo simulation 


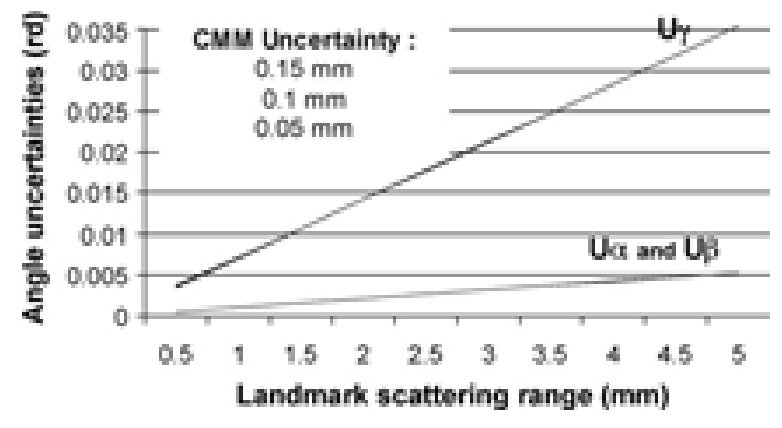

Fig. 8. Uncertainties evaluated by the analytical method.

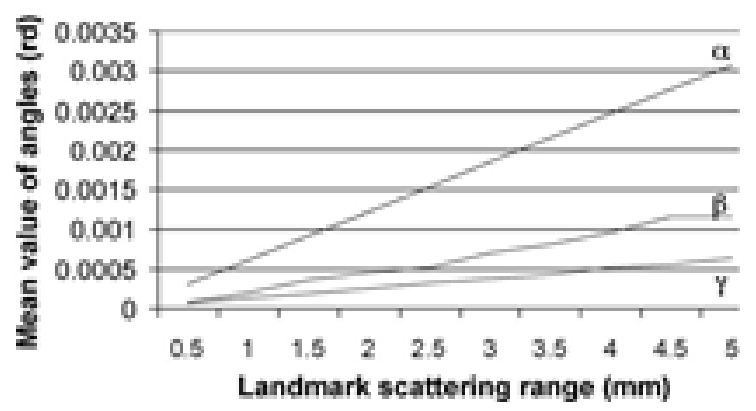

Fig. 9. Mean value of angles (CMM Uncertainty $=0.1 \mathrm{~mm}$ and Monte Carlo method).

Table 1

Experimental data characterizing the scattering of the landmarks probed by the surgeon [10].

\begin{tabular}{ll}
\hline Centre of femur & $7.5 \mathrm{~mm}$ \\
Medial femoral epicondyle & $6.2 \mathrm{~mm}$ \\
Lateral femoral epicondyle & $3.75 \mathrm{~mm}$ \\
Centre of tibia & $8 \mathrm{~mm}$ \\
Medial malleolus & $2 \mathrm{~mm}$ \\
Lateral malleolus & $6 \mathrm{~mm}$ \\
\hline
\end{tabular}

Table 2

Results (Monte Carlo simulations)

\begin{tabular}{lll}
\hline $\mathrm{a}$ mean & $\mathrm{b}$ mean & $\mathrm{g}$ mean \\
\hline $0.002 \mathrm{rad}$ & $0.004 \mathrm{rad}$ & $0.007 \mathrm{rad}$ \\
0.18 & 0.28 & 0.48 \\
$\mathrm{Ua}$ & $\mathrm{Ub}$ & $\mathrm{Ug}$ \\
\hline $0.007 \mathrm{rad}$ & $0.008 \mathrm{rad}$ & $0.031 \mathrm{rad}$ \\
0.48 & 0.58 & 1.88 \\
\hline
\end{tabular}

Table 3

Results (analytical method)

\begin{tabular}{lll}
\hline Ua & Ub & Ug \\
\hline $0.008 \mathrm{rad}$ & $0.008 \mathrm{rad}$ & $0.037 \mathrm{rad}$ \\
0.58 & 0.58 & 28 \\
\hline
\end{tabular}

nonlinearity of the equations which leads to non-symmetrical probability densities of the calculated results.

The quality of the TKA process is principally measured by the deviations of angles $b$ and $g$. In fact, excess valgus-varus angle $b$, often leads to premature failure of the prosthesis. Moreover, a large rotation angle $\mathrm{g}$ gives an un-aesthetic position of the patient foot. From now, the scattering of the landmarks probed by the surgeon was selected arbitrary. To get more realistic values of uncertainties calculations were also performed using experimental data published by Yau et al. 10] (Table 1).

The related enlarged uncertainties were thus calculated for the three angles $\mathbf{a}, \mathbf{b}$ and $\mathrm{g}$. Both values in radians and degrees are presented in Table 2 for the Monte Carlo simulation method and Table 3 for the analytical propagation. For the first estimation technique the bias of the mean values are also reported.

Both methods give close results. The maximal variation of the valgus-varus angle (b) is limited to 0.58 . The variation of the axial rotation angle $(\mathrm{g})$ is estimated to 28 .

\section{Conclusion}

This paper presents a methodology to determine the bias and uncertainty of the angles existing between the two implanted parts of knee prostheses after total arthroplasty.

The proposed method simulates the measurement process by a parametric virtual geometrical model of the anatomic skeleton. It permits constructing sets of measurements as acquired by a classical CAOS system.

A Monte Carlo simulation method and the classical analytical propagation technique of the GUM have been tested then to estimate the uncertainties of the reference frames defining the cutting planes for arthroplasty.

In the second method, the Jacobian matrix of the functions of interest is obtained by numerical derivation avoiding fastidious calculations. The two methods gave equivalent results. Hoverer the analytical approach has provided faster results than Monte Carlo simulations.

This study permitted to demonstrate than the uncertainties of the optical CMM have negligible effect on the final adjustment of the prosthesis. On the contrary, the scattering of the landmarks probed by the surgeon has significant influence. It leads to

deviations of 0.58 for the valgus-varus angle and 28 for the axial rotation.

\section{References}

[1] Williams DJ, Ratchev S, Chandra A, Hirani H (2006) The Application of Assembly and Automation Technologies to Healthcare Products. Annals of CIRP 55:617-642.

[2] Mitsuishi M, Sugita N, Fujiwara K, Abe N, Ozaki T, Suzuki M, Moriya H, Inoue T Kuramoto K, Nakashima Y, Tanimoto K (2007) Development of a Medical CAD/ CAM System for Orthopedic Surgery. Annals of CIRP 56:405-410.

[3] Mitsuishi M, Warisawa S, Sugita N (2004) Determination of the Machining Characteristics of a Biomaterial Using a Machine Tool Designed for Total Knee Arthroplasty. Annals of CIRP 53:107-112.

[4] Mitsuishi M, Warisawa S, Sugita N, Suzuki M, Moriya H, Hashizume H Fujiwara K, Abe N, Inoue H, Kuramoto K, Inoue T, Nakashima Y, Tanimoto K (2005) A Study of Bone Micro-Cutting Characteristics using a Newly Developed Advanced Bone Cutting Machine Tool for Total Knee Arthroplasty. Annals of CIRP 54:41-46

[5] Mitsuishi M, Warisawa S, Tajima F, Suzuki M, Tanimoto K, Kuramoto K (2003) Development of a 9 Axes Machine Tool for Bone Cutting. Annals of CIRF 52:323-328.

[6] Schwenke H, Siebert BRL, Waldele F, Kunzmann H (2000) Assessment of Uncertainties in Dimensional Metrology by Monte Carlo Simulation. Proposal of a Modular and Visual Software. Annals of CIRP 49:395-398.

[7] Balsamo A, Di Ciommo M, Mugno R, Rebaglia BI, Ricci E, Grella R (1999) Evaluation of CMM Uncertainty though Monte Carlo Simulations. Annals of CIRP 48:429-432.

[8] Sprauel JM, Linares JM, Bachmann J, Bourdet P (2003) Uncertainties in CMM Measurements, Control of ISO Specifications. Annals of CIRP 51:423-426.

[9] Guide to the Expression of Uncertainty in Measurement, (1993), First Edition. International Organization for Standardization.

[10] Yau WP, Leung A, Chiu KY, Tang WM, Ng TP (2005) Intraobserver Errors in Obtaining Visually Selected Anatomic Landmarks During Registration Proces: in Nonimage-based Navigation-assisted Total Knee Arthroplasty: A Cadaveric Experiment. Journal of Arthroplasty 20(5):591-601. 